\title{
Predictive factors for skeletal complications in hormone-refractory prostate cancer patients with metastatic bone disease
}

\author{
A Berruti', M Tucci', A Mosca', R Tarabuzzi', G Gorzegno', C Terrone', F Vana', G Lamanna', \\ M Tampellini', F Porpiglia', A Angeli', RM Scarpa' and L Dogliotti*, \\ 'Dipartimento di Scienze Cliniche e Biologiche Università degli Studi di Torino, Prostate Cancer Unit, Oncologia Medica, Urologia, Medicina Interna, \\ Azienda Ospedaliera San Luigi, Orbassano, Italy
}

\begin{abstract}
Factors predictive of skeletal-related events (SREs) in bone metastatic prostate cancer patients with hormone-refractory disease were investigated. We evaluated the frequency of SREs in 200 hormone-refractory patients consecutively observed at our Institution and followed until death or the last follow-up. Baseline parameters were evaluated in univariate and multivariate analysis as potential predictive factors of SREs. Skeletal-related events were observed in 86 patients (43.0\%), 10 of which (5.0\%) occurred before the onset of hormone-refractory disease. In univariate analysis, patient performance status $(P=0.002)$, disease extent (DE) in bone $(P=0.000 \mathrm{I})$, bone pain $(P=0.000 \mathrm{I})$, serum alkaline phosphatase $(P=0.000 \mathrm{I})$ and urinary $\mathrm{N}$-telopeptide of type one collagen $(P=0.000 \mathrm{I})$ directly correlated with a greater risk to develop SREs, whereas Gleason score at diagnosis, serum PSA, Hb, serum albumin, serum calcium, types of bone lesions and duration of androgen deprivation therapy did not. Both DE in bone (hazard ratio $(H R):$ I.I6, 95\% confidence interval $(\mathrm{Cl}): 1.07-1.25, P=0.000)$ and pain score (HR: I.I3, 95\% Cl: I.06-1.20, $P=0.000)$ were independent variables predicting for the onset of SREs in multivariate analysis. In patients with heavy tumour load in bone and great bone pain, the percentage of SREs was almost twice as high as ( 26 vs 52\%, $P<0.02)$ and occurred significantly earlier $(P=0.000)$ than SREs in patients with limited DE in bone and low pain. Bone pain and DE in bone independently predict the occurrence of SREs in bone metastatic prostate cancer patients with hormone-refractory disease. These findings could help physicians in tailoring the skeletal follow-up most appropriate to individual patients and may prove useful for stratifying patients enrolled in bisphosphonate clinical trials.
\end{abstract}

British Journal of Cancer (2005) 93, 633-638. doi: I 0.1038/sj.bjc.6602767 www.bjcancer.com

(C) 2005 Cancer Research UK

Keywords: prostate cancer; bone metastases; adverse skeletal events

Prostate cancer is the most commonly diagnosed malignancy among elderly men and the second most common cause of cancerrelated death in men in Italy and other European countries (De Angelis et al, 1997). In patients with prostate cancer, bone is by far the most common site of distant metastases. More than $80 \%$ of men with advanced disease will, in fact, eventually develop skeletal involvement. Bone metastases are devastating events in the natural history of the disease since they are often painful and cause skeletal complications such as pathologic fracture, vertebral collapse and spinal cord compression (Berruti et al, 2000; Bayley et al, 2001; Mundy, 2002). Androgen suppression by either orchiectomy or administration of luteinising hormone-releasing hormone analogs (LHRH-A) is the mainstay of treatment for advanced prostate cancer patients. This therapy frequently produces tumour shrinkage and improvement in symptoms, but it is not curative since almost all patients will eventually develop hormone-refractory disease. Once the disease becomes refractory to hormonal manipulation, the prognosis is dismal and the overall survival is about 13-16 months (Smaletz et al, 2002; Halabi et al, 2003).

\footnotetext{
*Correspondence: Professor L Dogliotti; E-mail: luigi.dogliotti@unito.it
} Received 24 May 2005; revised I4 July 2005; accepted 2 August 2005
We have previously observed that skeletal-related events (SREs) in prostate cancer patients are relatively rare as long as the disease is responsive to hormones, but they occur much more often when the tumour becomes hormone refractory (Berruti et al, 2000). Zoledronic acid, a potent bisphosphonate, significantly reduces the onset of skeletal complications in this patient population (Saad et al, 2002). Recent reports point out, however, that administration of this drug is not cost-effective (Reed et al, 2004). Other available treatment options include intensive supportive care, radiotherapy, second-line hormonal manipulations, cytotoxic chemotherapy and investigational agents (Goodin et al, 2002). In two recent randomised independent clinical trials, chemotherapy reportedly improved response rate and survival (Petrylak et al, 2004; Tannock et al, 2004). However, because the survival benefit is modest, treatment options for bone metastatic patients with hormonerefractory disease are mainly palliative.

Since skeletal complications are the major causes of diminished quality of life in this patient population (Groot et al, 2003), the identification of baseline factors predictive for these adverse events may be a valuable aid in tailoring to each patient the most appropriate preventive measures and in potentially avoiding unnecessary and costly treatments.

In advanced prostate cancer, the basic factors contributing to skeletal-related morbidity may be broken down loosely into those 
related directly to the metastases and those of iatrogenic nature. Androgen deprivation therapy causes osteoporosis and adverse skeletal events independently of disease response and in the absence of skeletal involvement (Daniell et al, 2000; Smith, 2003). The frequency of adverse skeletal events induced by androgen deprivation therapy depends mainly on the duration of therapy. Long-lasting androgen deprivation therapy makes the skeleton more fragile and may contribute to skeletal morbidity even in the hormone-refractory phase of the disease.

In this study, the frequency of SREs was evaluated in a series of hormone-refractory prostate cancer patients with metastatic bone disease prospectively treated and followed at the Prostate Cancer Unit of the Azienda Ospedaliera San Luigi di Orbassano, Italy. In a previous publication on the first 112 cases (Berruti et al, 2000), we identified several predictive factors, including serum alkaline phosphatase (ALP), urinary deoxy-pyridinoline and disease extent (DE) in bone. Here, we tested by univariate and multivariate analyses a number of additional predictive factors for SREs, including validated prognostic parameters, urinary $\mathrm{N}$-telopeptide of type one collagen (NTx) and duration of androgen deprivation therapy.

\section{PATIENTS AND METHODS}

\section{Patients}

Eligible patients were required to have histologically proven adenocarcinoma of the prostate with metastatic bone disease that progressed to LHRH-A administration with or without antiandrogens. Disease progression was defined as a rise in prostate-specific antigen (PSA) levels ( $\geqslant 50 \%$ over the nadir obtained during androgen deprivation) on two consecutive measurements performed at least 2 weeks apart or a new lesion on bone scan and/ or an increase in the size of a measurable lesion on a computed tomographic (CT) scan of the abdomen/pelvis or chest. The time from diagnosis of hormone refractoriness to enrolment in the study should have been not greater than 2 months.

Patients previously treated with LHRH-A alone underwent total androgen ablation (obtained by adding an antiandrogen: flutamide or bicalutamide). They entered the study if they showed further evidence of disease progression after at least 4 weeks of combined therapy. Patients previously treated with total androgen ablation were required to undergo antiandrogen withdrawal. They were required to be off all antiandrogens for at least 4 weeks, with further evidence of disease progression after cessation of antiandrogen treatment. Serum testosterone was routinely measured in all patients showing PSA progression under LHRH-A since 1997. From this date onwards, testosterone inhibition (defined as serum levels $<50 \mathrm{ng} \mathrm{dl}^{-1}$ ) was added to the criteria for hormone refractoriness. Further inclusion criteria comprised an Eastern Cooperative Oncology Group (ECOG) performance status (PS) of $0-3$ and normal renal and hepatic function. Patients were excluded from the study if they had severe uncontrolled comorbidity, second malignancies, pretreatment with bisphosphonates, radiotherapy or radionuclide therapy for palliation of bone pain, and second-line antineoplastic treatment. At study entry, all patients underwent physical examination, including bone pain assessment and neurological examination, routine blood chemistry studies, serum PSA, serum ALP, bone scan followed by radiological confirmation of hot spots, chest X-ray and whole abdomen CT. Urinary NTx was measured in the last 103 patients. When scintigraphy was positive and X-ray films were negative for bone lesions, a CT study was performed. The whole skeleton was arbitrarily divided into the following areas: skull, cervical, dorsal, lumbar spine + sacrum, right leg, left leg, right arm, left arm, right ribs, left ribs, sternum, right scapula and clavicula, left scapula and clavicula, right pelvis and left pelvis. Disease extent in bone was calculated as the sum of involved areas. In order to limit the distribution of this variable, patients with $\geqslant 9$ segments were scored as 9. Baseline bone pain was evaluated using a validated pain questionnaire, as previously reported (Berruti et al, 2002). Questionnaire items included performance status, analgesic consumption and mobility, as measured on a pain score of $0-$ 19. All patients subsequently received second-line treatment consisting of endocrine therapy, chemotherapy, radiotherapy and radionuclide therapy, as indicated in association with the best palliative care. Physical and bone pain reassessment, routine blood chemistry studies, bone markers and serum PSA were repeated at least every 3 months. On detection of increased PSA, worsening bone pain and/or impaired mobility, patients underwent bone scanning followed by radiological confirmation of hot spots. When pathological or impending fracture was diagnosed, patients were referred to an orthopaedic surgeon. When deformity of 1 or more vertebral bodies was detected or clinical evidence of spinal cord compression was suspected, whole spine magnetic resonance imaging (MRI) was performed to direct decision-making whether to perform surgery or radiotherapy and/or prescribe spinal orthosis. The study was approved by the Institutional Review Board of our hospital. All patients were required to give written informed consent before registration.

\section{Study end points}

Skeletal-related events were defined as vertebral body collapse requiring spinal orthosis, spinal cord compression, vertebral and nonvertebral pathological fractures, symptomatic hypercalcemia, symptomatic hypocalcemia. Skeletal-related events also included surgery and/or radiotherapy delivered to bone to stabilise or prevent pathologic fractures or areas of spinal cord compression. Neither radiation therapy nor radionuclide therapy for pain relief nor change in antineoplastic therapy to treat bone pain were considered SREs. Survival duration was defined as the time between the diagnosis of hormone-refractory disease and death. The date of hormone refractoriness was defined as the date of the first rise in PSA or, in the rare cases of no PSA elevation, the date of diagnosis of disease progression in skeletal or extraskeletal sites. Patients were censored if they were known to be alive or were lost to follow-up. The time to the onset of skeletal complications was calculated as the time between the diagnosis of hormone-refractory disease and the occurrence of the first SRE or death, whichever event occurred first. Patients were censored if they were alive and free of SREs. If the first SRE occurred before the onset of hormone refractoriness, it was not computed in the analysis.

\section{Biochemical measurements}

Early morning spot urine specimens were obtained to measure creatinine and $\mathrm{N}$-telopeptide; blood samples were drawn to assess calcium, albumin (ALB), PSA, total ALP, lactate dehydrogenasis (LDH) and haemoglobin (Hb). $\mathrm{N}$-telopeptide of type one collagen was measured using a commercial kit (Osteomark, Ortho-Clinical Diagnostics, Rochester, NY, USA), blood biochemical parameters were measured using automated procedures (Architect, Abbott).

\section{Statistical analysis}

Differences in proportion were determined using the $\chi^{2}$ test. Correlations between continuous variables were performed using the nonparametric Spearman test. Survival curves were estimated using the Kaplan-Meier method. Unadjusted differences in these estimates were tested using the log-rank test. A univariate Cox proportional hazards model was used to identify all statistically significant predictors of SREs and death. A multivariate Cox proportional hazards model was used to identify independent variables predictive of the onset of SREs. Only those variables 
found to be significant on univariate analysis were included in a multivariate proportional hazards model. Among these, serum ALP and urinary NTx had right skewed distributions and were modelled using log transformation. Two analyses were performed, one on overall cases without including NTx and another on the 103 patients having NTx measured. The latter analysis should be considered as explorative. All reported $P$-values were two-sided; $P$ values $<0.05$ were considered statistically significant. Statistical computation was performed using the SPSS for Windows software package.

\section{RESULTS}

\section{Patients}

From July 1990 to January 2003, 200 consecutive patients with newly diagnosed hormone-refractory prostate cancer meeting the eligibility criteria were enrolled in the present study. Patient characteristics are depicted in Table 1. Urinary NTx was available for the last 103 patients, while in the first patients bone resorption was measured by urinary deoxypyridinoline. Treatment for hormone-refractory disease was chemotherapy in 74 patients (estramustine alone, etoposide + estramustine, epirubicin, docetaxel) and steroids plus supportive care in the remaining 126 cases. Previous LHRH-A administration was not interrupted in any cases. Bone pain was present in 177 patients (88.5\%); $72(36.0 \%)$ necessitated radiotherapy and or radionuclide therapy to control pain symptoms; 32 (16.0\%) also received single-dose pamidronate, as previously reported (Berruti et al, 2002). As expected, a significant correlation was found between $\mathrm{DE}$ in bone and either ALP serum levels (Spearman R 0.51, $P<0.01$ ) or NTx urinary values (Spearman $\mathrm{R} 0.40, P<0.01$ ).

A total of 86 patients $(43.0 \%)$ experienced skeletal complications, including vertebral collapse in $41(20.5 \%)$, fractures in 25

Table I Patient characteristics

\begin{tabular}{|c|c|}
\hline No & 200 \\
\hline Age median (year) (range) & $73(52-92)$ \\
\hline \multicolumn{2}{|l|}{ ECOG PS } \\
\hline 0 & $42(21.0 \%$ \\
\hline I & $91(45.5 \%)$ \\
\hline $2-3$ & $67(33.5 \%)$ \\
\hline \multicolumn{2}{|l|}{ Gleason score at diagnosis (196 patients) } \\
\hline$<8$ & $95(48.5 \%)$ \\
\hline$\geqslant 8$ & $101(51.5 \%)$ \\
\hline \multicolumn{2}{|l|}{ Previous endocrine therapies } \\
\hline LHRH-A & | 82 (91.0\%) \\
\hline LHRH-A+antiandrogens & $18(9.0 \%)$ \\
\hline PSA (ng ml $\left.{ }^{-1}\right)$ (198 patients) $^{\mathrm{a}}$ & | $18.0(0.1-9000.0)$ \\
\hline Alkaline phosphatase $\left(\mathrm{UI}^{-1}\right)$ (I 86 patients) $^{\mathrm{a}}$ & $165.0(47.0-6000.0)$ \\
\hline NTx (nmol mmol ${ }^{-1}$ creatinine) (I03 patients) ${ }^{a}$ & $96.0(11.6-662.0)$ \\
\hline $\mathrm{Ca}\left(\mathrm{mmol} \mathrm{I}^{-1}\right)\left(184\right.$ patients) ${ }^{\mathrm{a}}$ & $2.35(1.91-4.20)$ \\
\hline $\mathrm{Hb}\left(\mathrm{g} \mathrm{dl}^{-1}\right)(182 \text { patients})^{\mathrm{a}}$ & $12.1(7.3-15.6)$ \\
\hline Albumin $\left(\mathrm{g} \mathrm{dl}^{-1}\right)$ (I70 patients) $^{\mathrm{a}}$ & $3.6(1.9-6.7)$ \\
\hline $\mathrm{LDH}\left(\cup \mathrm{I}^{-1}\right)(\mathrm{I} 78 \text { patients })^{\mathrm{a}}$ & $405(|36-30| 1)$ \\
\hline Androgen deprivation duration (months) (I 88 patients) ${ }^{\mathrm{a}}$ & $24.5(2.03-228.0)$ \\
\hline Disease extent in bone (number of sites) (198 patients) ${ }^{a}$ & $6(1-9$ or more $)$ \\
\hline Pain score (200 patients) ${ }^{a}$ & $5(0-16)$ \\
\hline \multicolumn{2}{|l|}{ Types of bone lesions (190 patients) } \\
\hline Lytic & $14(7.4 \%)$ \\
\hline Mixed & $42(22.1 \%)$ \\
\hline Blastic & $134(70.5 \%)$ \\
\hline
\end{tabular}

aData are median (range).
(12.5\%) and spinal cord compression in 20 (10.0\%). Skeletal fractures involved the vertebrae (seven patients), the ribs (six patients), the femur (six patients), the scapula (one patient), the pelvis (three patients), the humerus (one patient), the vertebrae and ribs (one patient). Two patients developed symptomatic hypercalcemia and one patient symptomatic hypocalcemia. Both metabolic adverse events occurred concomitantly with other SREs and were not computed separately.

All patients who experienced SREs received radiation therapy; 13 required surgery to bone. Additional SREs occurring in patients who had already experienced an adverse skeletal event were not considered in the analysis. In $10(5.0 \%)$, the first adverse SRE preceded the onset of hormone refractory disease; in $76(38.0 \%)$ it occurred afterwards. In those cases where SREs occurred before hormone refractoriness, the median duration of androgen deprivation therapy was 59.9 months (range 19.3-228.0) and 24.9 months in those where it did not (range 3.0-212.0, $P=0.004$ ). In the patients who experienced SREs in the hormone-refractory phase of the disease, the median time to the onset of adverse events was 7 months (range 1-39). At the last follow-up on 15 August 2004, 180 patients $(90.0 \%)$ had died (median survival 14.5 months); 102 of which (56.7\%) without experiencing SREs. Out of 20 patients, 12 still alive were SRE-free, five of which were alive more than 4 years since the date of the onset of hormonerefractory disease.

\section{Skeletal-related events according to baseline characteristics}

The proportion of patients with at least one SRE according to baseline parameters and the univariate relative risks for the onset of SREs and death is shown in Table 2. Skeletal-related events occurring before the onset of hormone-refractory disease were not considered in the analysis. Disease extent in bone, bone pain, serum ALP and urinary NTx directly correlated with a higher risk of developing adverse skeletal events over time (either in terms of proportion of SREs or in terms of relative risk). Analysis of Gleason score, serum PSA, Hb, serum albumin, serum calcium, types of bone lesions and duration of androgen deprivation therapy did not show any significant relationship between these parameters and the occurrence of SREs. Performance status failed to be significantly correlated with the frequency of SREs, while it was significantly related with a higher risk of experiencing an SRE over time.

Those variables attaining statistical significance in the univariate analysis were included in the multivariate analysis. In the first multivariate analysis of all cases, both $\mathrm{DE}$ in bone and bone pain were independent variables predicting for SREs, while ALP and patient performance status did not enter the model (Table 3 ). In the multivariate analysis on patients with NTx assessed alone, DE in bone and bone pain maintained an independent predictive role, while NTx failed to enter the model (HR: 1.13 , 95\% CI: $0.71-1.77$ ).

Disease extent in bone and bone pain (both considered dichotomous variables as outlined in Table 1) were combined to generate three patient subgroups: patients with DE in $\leqslant 6$ sites and a pain score of $<5$ (DE-/Pain-), patients with $\mathrm{DE}$ in $>6$ sites and a pain score of $\geqslant 5($ Pain $+/ D E+)$ and patients with either of these predictors (DE-/Pain + or $\mathrm{DE}+/$ Pain-). The distribution of SREs in patients with Pain $+/ D E+$ was double that of patients with Pain-/DE- (26.2 vs 52.5\%, respectively, $P=0.016)$, while the SRE frequency in patients with either of these two predictors was intermediate (36.0\%). Analysis of the time to onset of first SRE also showed a time-dependent pattern (Figure 1). Patients with heavy tumour load in bone and great bone pain $($ Pain $+/ D E+)$ developed SREs significantly earlier than those with limited DE in bone and low pain (Pain-/DE-) (RR: 1.63, 95\% CI: 1.32-2.00). Again, patients with either predictor showed an intermediate pattern. 
Table 2 Proportion of adverse skeletal related events (SREs), relative risks (RRs) and 95\% confidence intervals (Cls) associated with SREs or death

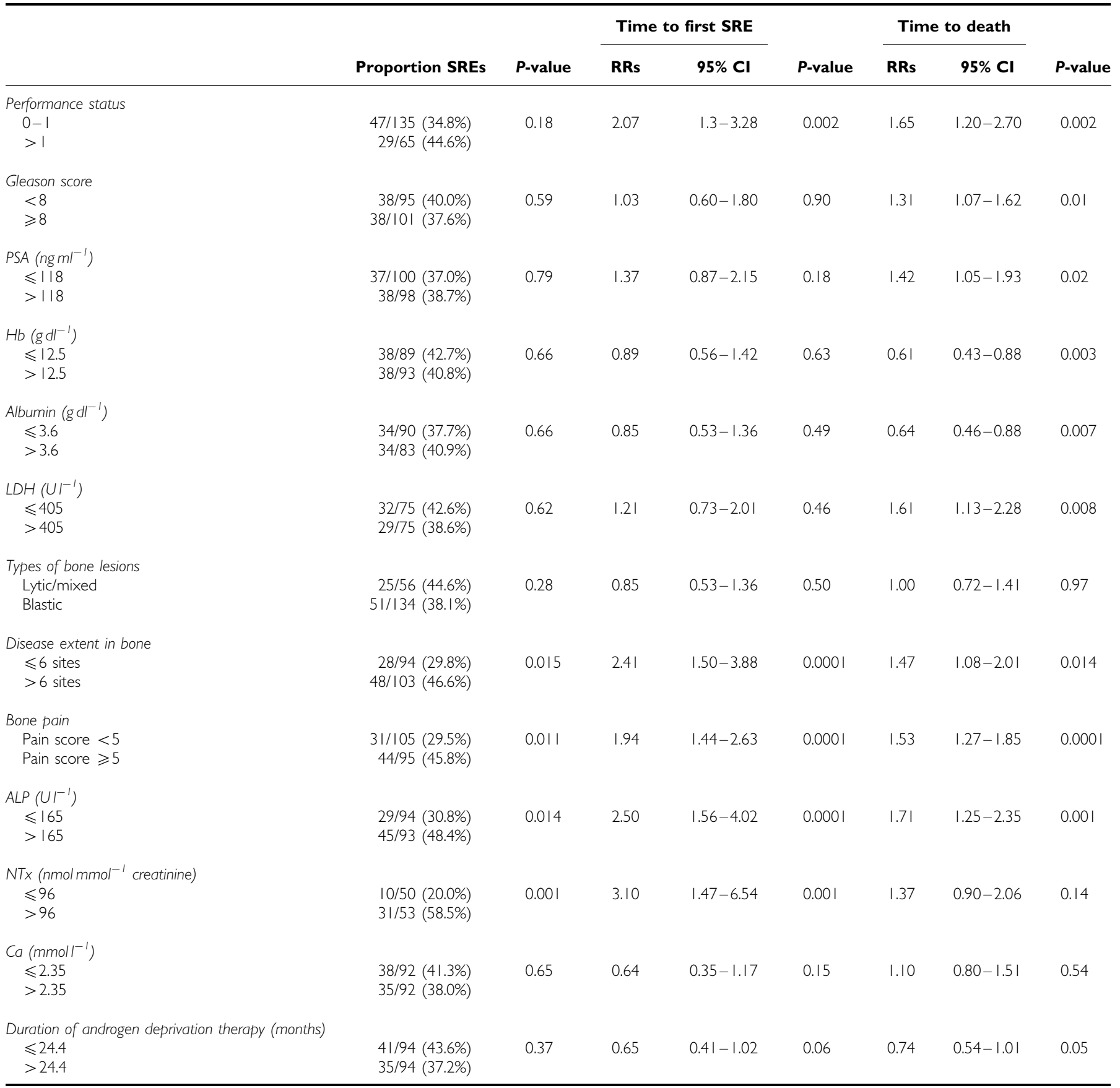

Table 3 Independent factors predictive for adverse skeletal events according to a multivariate Cox model

\begin{tabular}{lccc}
\hline Variables & $\begin{array}{c}\text { Hazard } \\
\text { ratio }\end{array}$ & $\begin{array}{c}\mathbf{9 5 \%} \\
\text { confidence } \\
\text { intervals }\end{array}$ & P-value \\
\hline $\begin{array}{l}\text { Variables not in the model } \\
\quad \text { Alkaline phosphatase }\end{array}$ & 1.11 & $0.84-1.48$ & 0.45 \\
$\quad \begin{array}{l}\text { Performance status } \\
\text { Variables in the model }\end{array}$ & 1.22 & $0.70-2.12$ & 0.47 \\
$\quad$ Number of metastatic sites & 1.16 & $1.07-1.25$ & \\
$\quad$ Pain score & 1.13 & $1.06-1.20$ & 0.000 \\
\hline
\end{tabular}

All validated prognostic parameters such as Gleason score, serum albumin, serum ALP, LDH, Hb and PSA were significantly related with poor survival; in addition, DE in bone also showed a negative relationship (Table 2 ).

\section{DISCUSSION}

Adverse skeletal events often lead to a deterioration in the quality of life of prostate cancer patients (Weinfurt et al, 2005). In our series of prospectively followed bone metastatic patients with hormone-refractory disease, $43 \%$ experienced SREs, with $5 \%$ occurring before and $38 \%$ after the onset of hormone-refractory disease. The time to onset of the first adverse event in the 


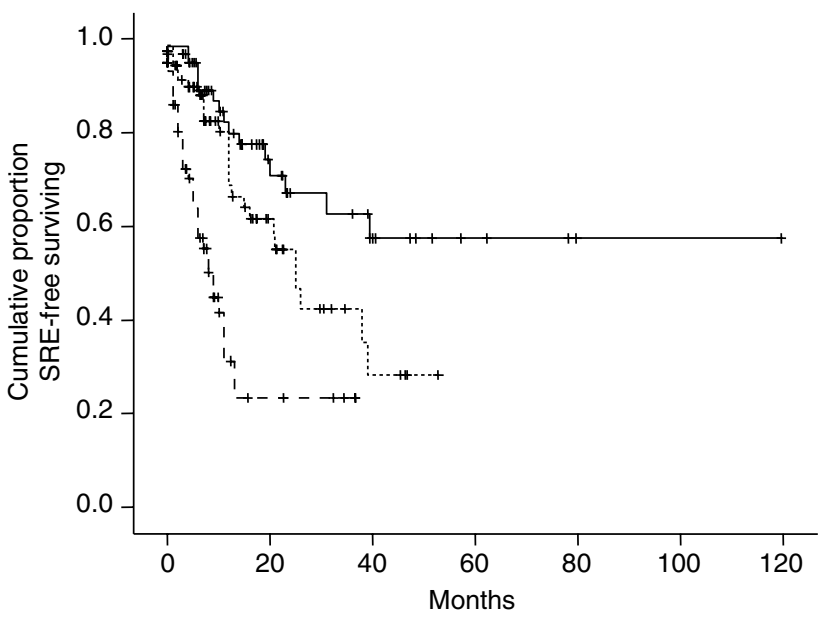

Figure I Time to the onset of SRE in patients with low pain and limited disease extent in bone (Pain-/DE-) (_- vs patients with great bone pain and heavy tumour load in bone (Pain $+/ D E+)(---\cdot)$, vs patients with either of these two predictors (...........).

hormone-refractory phase, although only 7 months on average, was distributed over a wide range (1-39 months). In all, $90 \%$ of patients died, $58.8 \%$ of which died of disease without experiencing an SRE. Among those still alive at the last follow-up appointment, five were still SREs-free more than 4 years since the date of hormone refractoriness.

The first comment to this study is that many patients are expected to develop SREs in the hormone-refractory phase of the disease, but they constitute a minority of those potentially at risk. In this respect, the hormone-refractory patients were a heterogeneous population, since SREs occurred within a few months in many patients or sometimes even years later in others.

Knowing whether the chance to develop SREs is high or low and whether an SRE may occur within months or years is useful for physicians when planning the frequency of follow-up investigations and guiding decisions about the choice of treatment. In addition, the identification of predictive factors for developing SREs may be a valuable aid in designing studies to test bone resorption inhibitors that can prevent adverse skeletal events, since an imbalance in these factors when comparing study arms could lead to biased results.

Zoledronic acid administration in hormone-refractory prostate cancer patients with metastatic bone disease has been demonstrated to significantly reduce the frequency of SREs. No patients in our series received zoledronic acid, but 31 had been treated with a single dose of pamidronate, another bisphosphonate (Berruti et al, 2002). Since pamidronate had failed to reduce SREs in the prostate cancer population (Lipton et al, 2002), these patients were not removed from the analysis.

A recent study pointed out that zoledronic acid administration in bone metastatic prostate cancer patients with hormonerefractory disease is not cost-effective since the costs of administering the drug outweigh the savings on the cost of medical care linked to the reduction in SREs (Reed et al, 2004). We believe, however, that the use of this drug might be more convenient if it were administered only to patients at risk of developing SREs.

Among the skeletal related parameters considered in the present study as predictive factors for SREs, disease estent in bone, bone pain, serum ALP and urinary NTx displayed a significant association in univariate analysis, while serum calcium and type of bone lesions did not. In a previous paper, we reported that urinary deoxypyridinoline, a bone resorption marker, showed a significant correlation with the probability to develop SREs
(Berruti et al, 2000). In the present study, NTx also showed a similar relationship. $\mathrm{N}$-telopeptide of type one collagen has recently been demonstrated to be an independent predictor of SREs in prostate cancer patients and those with non-small-cell lung cancer or other solid tumours (Brown et al, 2005). Our data are in line with these results. Unfortunately, this marker failed to be an independent predictor when adjusted for $\mathrm{DE}$ in bone. The relationship between NTx with DE in bone could provide a possible explanation for this failure. $\mathrm{N}$-telopeptide of type one collagen, however, was available in about $50 \%$ of cases, so that caution should be taken when interpreting data coming from a subgroup analysis. Serum calcium is notoriously tightly regulated and so does not reflect the bone matrix break-down. This is probably the reason why it failed to show a predictive role in our series. The absence of a relationship between the type of bone lesions and the occurrence of SREs is somewhat surprising. It should be noted, however, that the small number of patients with lytic/mixed bone lesions may have limited the power of the analysis.

The multivariate analysis identified bone pain and $\mathrm{DE}$ in bone as two independent parameters predictive for the occurrence of SREs. By combining these two variables, dichotomised at median value, we defined three patient subgroups. The group with low pain score and limited DE in bone had a low incidence of SREs, whereas the group with high pain score and extensive tumour load in bone had a high incidence of SREs. The incidence of SREs in the patients group with either of these two predictors was intermediate. The time-to-first-SRE curves show that patients with heavy tumour load in bone and high pain score developed SREs much earlier than those with less tumour load and bone pain. This observation comes somewhat expectedly but has never been reported before. Disease extent in bone was found to be predictive for spinal cord compression in a small study on 68 cases, 61 of which had hormone-refractory disease (Bayley et al, 2001), thus confirming our observations. In the same study, however, the authors reported a direct relationship between the occurrence of spinal cord compression and the duration of androgen deprivation therapy, a finding that contrasts with our results.

Recognised prognostic factors such as Gleason score at diagnosis, Hb, LDH, PS and albumin (Smaletz et al, 2002; Halabi et $a l, 2003$ ) were confirmed as being significantly related with poor survival but failed to be predictive for SREs; in addition, some factors predictive for SREs were also negative prognostic parameters. By combining prognostic parameters (Smaletz et al, 2002; Halabi et al, 2003) with predictive factors of SREs, it may be possible to identify a patient subset with a higher likelihood of dying before the onset of SREs in which bisphosphonate therapy could be potentially avoided.

In our series, the duration of androgen deprivation therapy significantly correlated with the occurrence of SREs in the hormone-sensitive phase of the disease, thus confirming its causative role (Daniell et al, 2000; Oefelein et al, 2002), but failed to show a predictive role when the disease become hormone refractory. In this study, benign SREs cannot be distinguished from malignant SREs, which may be an obstacle to assessing the real impact of iatrogenic SREs. This issues needs to be addressed in future studies. Noteworthy, however, is that the majority of SREs were distributed among patients with heavy tumour load in bone. These data suggest that in the hormone-refractory phase of the disease bone loss due to tumour-induced osteolysis prevail over an iatrogenic effect.

In a large series of men with prostate carcinoma prospectively followed after initiation androgen deprivation therapy until death, the duration of hormone therapy conferred an independent risk for SREs (Krupski et al, 2004). In this study, however, only skeletal fractures were considered as SREs, and no distinction was made between SREs occurring before or after the onset of hormone refractory disease. 
In conclusion, prostate cancer patients with hormone-refractory disease are generally more likely to die of the disease than to develop SREs. Our study shows that bone pain intensity and DE in bone, two variables easily available in clinical routine, independently predict the occurrence of SREs in this patient population. By combining these parameters we found patient subsets with a different risk of SREs. These findings have several implications: (1) they could help physicians in planning the most appropriate skel-

\section{REFERENCES}

Bayley A, Milosevic M, Blend R, Logue J, Gospodarowicz M, Boxen I, Warde P, McLean M, Catton C, Catton P (2001) A prospective study of factors predicting clinically occult spinal cord compression in patients with metastatic prostate carcinoma. Cancer 92: 303-310

Berruti A, Dogliotti L, Bitossi R, Fasolis G, Gorzegno G, Bellina M, Torta M, Porpiglia F, Fontana D, Angeli A (2000) Incidence of skeletal complications in patients with bone metastatic prostate cancer and hormone refractory disease: predictive role of bone resorption and formation markers evaluated at baseline. J Urol 164(4): 1248-1253

Berruti A, Dogliotti L, Tucci M, Tarabuzzi R, Guercio S, Torta M, Tampellini M, Dovio A, Poggio M, Scarpa RM, Angeli A (2002) Metabolic effects of single-dose pamidronate administration in prostate cancer patients with bone metastases. Int J Biol Markers 17(4): 244-252

Brown JE, Cook RJ, Major P, Lipton A, Saad F, Smith M, Lee KA, Zheng M, Hei YJ, Coleman RE (2005) Bone turnover markers as predictors of skeletal complications in prostate cancer, lung cancer, and other solid tumors. J Natl Cancer Inst 97(1): 59-69

Daniell HW, Dunn SR, Ferguson DW, Lomas G, Niazi Z, Stratte PT (2000) Progressive osteoporosis during androgen deprivation therapy for prostate cancer. J Urol 163(1): $181-186$

De Angelis R, Capocaccia R, Verdecchia A (1997) Estimative relative survival of Italian cancer patients from sparse cancer registries data. Tumori 83: $33-38$

Goodin S, Rao KV, Di Paola RS (2002) State-of-the art treatment of metastatic hormone-refractory prostate cancer patients. Oncologist 7: $360-370$

Groot MT, Boeken Kruger CG, Pelger RC, Uyl-de Groot CA (2003) Costs of prostate cancer, metastatic to the bone, in the Netherlands. Eur Urol 43(3): $226-232$

Halabi S, Small EJ, Kantoff PW, Kattan MW, Kaplan EB, Dawson NA, Levine EG, Blumenstein BA, Vogelzang NJ (2003) Prognostic model for predicting survival in men with hormone refractory metastatic prostate cancer. J Clin Oncol 21: $1232-1237$

Krupski TL, Smith MR, Chan Lee W, Pashos CL, Brandman J, Wang Q, Botteman M, Litwin MS (2004) Natural history of bone complications in men with prostate carcinoma initiating androgen deprivation therapy. Cancer 101(3): $541-549$ etal follow-up for individual patients; (2) they suggest future trials aimed at delaying zoledronic acid administration in patients with low risk of developing SREs and/or avoiding drug delivery in patients with a high probability of dying of disease before the occurrence of SREs (ie patients with very limited disease in bone and concomitant visceral metastases). Finally, our results may prove useful for stratifying patients enrolled in clinical trials for testing the efficacy of new bone resorption inhibitors to prevent SREs.

Lipton A, Small E, Saad F, Gleason D, Gordon D, Smith M, Rosen L, Kowalski MO, Reitsma D, Seaman J (2002) The new bisphosphonate, Zometa (zoledronic acid), decreases skeletal complications in both osteolytic and osteoblastic lesions: a comparison to pamidronate. Cancer Invest 20(Suppl 2): $45-54$

Mundy GR (2002) Metastasis to bone: causes, consequences and therapeutic opportunities. Nat Rev Cancer 2(8): 584-593

Oefelein MG, Ricchiuti V, Conrad W, Resnick MI (2002) Skeletal fractures negatively correlate with overall survival in men with prostate cancer. J Urol 168(3): $1005-1007$

Petrylak DP, Tangen C, Hussain MHA, Lara Jr PN, Jones JA, Taplin ME, Burch PA, Berry D, Moinpour C, Kohli M, Benson MC, Small EJ, Raghavan D, Crawford ED (2004) Docetaxel and Estramustine compared with mitoxantrone and prednisone for advanced refractory prostate cancer. N Engl J Med 351: $1513-1520$

Reed SD, Radeva JI, Glendenning GA, Saad F, Schulman KA (2004) Costeffectiveness of zoledronic acid for the prevention of skeletal complications in patients with prostate cancer. J Urol 171(4): 1537-1542

Saad F, Gleason DM, Murray R, Tchekmedyian S, Venner P, Lacombe L, Chin JL, Vinholes JJ, Goas JA, Chen B, Zoledronic Acid Prostate Cancer Study Group (2002) A randomized, placebo-controlled trial of zoledronic acid in patients with hormone-refractory metastatic prostate carcinoma. J Natl Cancer Inst 94(19): 1458-1468

Smaletz O, Scher HI, Small EJ, Verbel DA, McMillan A, Regan K, Kelly WK, Kattan MW (2002) Nomogram for overall survival of patients with progressive metastatic prostate cancer after castration. J Clin Oncol 20: $3972-3982$

Smith MR (2003) Management of treatment-related osteoporosis in men with prostate cancer. Cancer Treat Rev 29(3): 211-218

Tannock IF, de Wit R, Berry WR, Pluzanska A, Chi KN, Oudard S, Theodore C, James ND, Turesson I, Rosenthal MA, Eisenberger MA, TAX 327 Investigators (2004) Docetaxel plus prednisone or mitoxantrone plus prednisone for advanced prostate cancer. N Engl J Med 351: 1502-1512

Weinfurt KP, Li Y, Castel LD, Saad F, Timbie JW, Glendenning GA, Schulman KA (2005) The significance of skeletal-related events for the health-related quality of life of patients with metastatic prostate cancer. Ann Oncol 16: 579-584 